\title{
Dark Matter as the Gravitized Vacuum: A Brief Note and Experimental Proposal
}

\author{
Eugene Terry Tatum \\ 760 Campbell Ln. Ste. 106 \#161, Bowling Green, KY, USA \\ Email: ett@twc.com
}

How to cite this paper: Tatum, E.T. (2018) Dark Matter as the Gravitized Vacuum: A Brief Note and Experimental Proposal. Journal of Modern Physics, 9, 2342-2345. https://doi.org/10.4236/jmp.2018.913148

Received: October 12, 2018

Accepted: November 17, 2018

Published: November 20, 2018

Copyright $\odot 2018$ by author and Scientific Research Publishing Inc. This work is licensed under the Creative Commons Attribution International License (CC BY 4.0).

http://creativecommons.org/licenses/by/4.0/

\section{c) (i) Open Access}

\begin{abstract}
The gravitized vacuum is operationally defined as the vacuum of space sufficiently close to any gravitating massive body, or collection of bodies, such that its gravitational energy field strength and density are observed, or expected, to be greater than that of deep intergalactic space. It is hypothesized that the contributions to gravitational lensing and excess galactic/peri-galactic rotational inertia currently attributed to dark matter could be predictable effects of increased energy density, with corresponding mass effects, of a gravitized vacuum acting in the manner of a near-absolute zero superfluid. This hypothesis should be testable by an earth-based laboratory with the apparatus and procedure described herein.
\end{abstract}

\section{Keywords}

Dark Matter, Gravitized Vacuum, Gravitational Lensing, Vacuum Energy, Emergent Gravity, Superfluidity, Gravitational Theory, General Relativity, Cosmology

\section{Introduction and Background}

It is well-known that vacuum regions within and around galaxies refract distant starlight and exhibit gravitational effects currently attributed to invisible matter. The first property, "gravitational lensing," is largely attributed to this "dark matter" (in conjunction with the visible matter). It is also well-known that, despite the recent apparent completion of the standard particle model with the discovery of the Higgs boson, current proposals for the nature of a dark matter particle do not appear to fit within the standard model. After numerous failed creative attempts to capture and characterize particulate dark matter, non-particulate proposals for the observational phenomena mentioned above are now being taken more seriously [1] [2] [3] [4] [5]. Eric Verlinde's "emergent gravity" 
theory, in particular, now even appears to have some observational support with respect to dark matter [6] [7]. An up-to-date review [8] published in the August 2018 issue of Scientific American summarizes the remaining viable theories of dark matter. Among them is the tantalizing possibility that dark matter observations could be the result of a superfluid phase transition within the vacuum [9].

There is nothing in the current observations of dark matter which mandates that it be particulate in nature. Gravitational lensing was a firm prediction of general relativity long before the first observations attributed to dark matter. In fact, one can consider the famous 1919 eclipse observations of the bending of starlight within the vacuum bordering the sun as observational confirmation of gravitational lensing. Einstein's geometrical description of curved space-time within the vacuum was sufficient to predict this effect. In this respect, one can view general relativity as a mathematical treatise which accurately describes the light-bending (i.e., refractive) gravitational effects on photons within a medium in which a gravitating body is embedded. Einstein's geometrical approach described a smoothly-modulated (i.e., non-particulate) energy field within the medium. Furthermore, given the $E=m c^{2}$ equivalency, any such energized medium can be considered to have greater mass density (i.e., gravitating effect) than the same medium at its lowest gravitational energy (i.e., zero gravity) state. Accordingly, it is the purpose of this paper to present a new theory of dark matter observation, and an experimental apparatus for its possible detection.

\section{Theory of Dark Matter as the Gravitized Vacuum}

Based upon the foregoing considerations, the "gravitized vacuum" is operationally defined as the vacuum of space sufficiently close to any gravitating massive body, or collection of bodies, such that its gravitational energy field strength and density are observed, or expected, to be greater than that of deep intergalactic space. Furthermore, it is proposed as a hypothesis that all observations attributed to dark matter are predictable effects of the increased energy density, with corresponding mass effects, of the gravitized vacuum. Specifically, that the dark matter gravitational lensing contribution is due to predictable density differences between the galactic/peri-galactic vacuum (greater density) and the deep intergalactic vacuum (lesser density). Furthermore, that the excess galactic gravitational inertia attributed to dark matter is due to previously unaccounted-for increased mass density of the gravitized galactic/peri-galactic vacuum.

\section{Experimental Proposal to Measure a Gravitized Vacuum}

The reader is referred to schematic Figure 1 for the following description of the experimental apparatus. In panel A, a large hollow metallic sphere evacuated to a near-total vacuum state is suspended in mid-air from a sensitive scale in a vibration-dampened laboratory. The experimental apparatus is chilled as close as possible to $2.725 \mathrm{~K}$. The weight of the sphere is carefully measured in this "pre-gravitized" state. In panel B, the weight of the suspended sphere is measured 


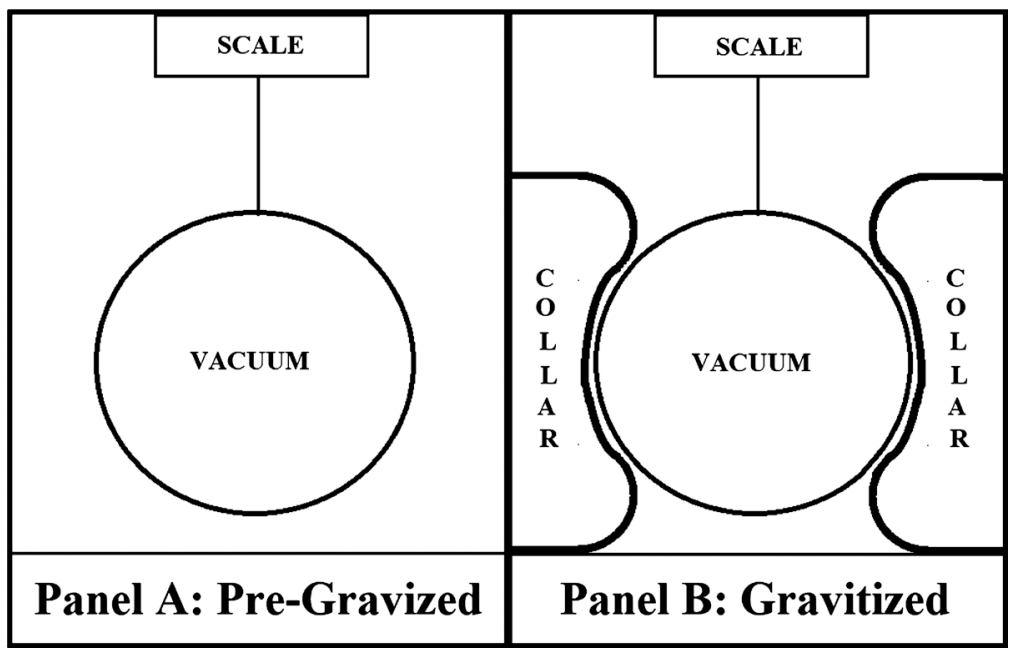

Figure 1. Experimental Apparatus for Pre-Gravitized and Gravitized Measurements.

again after the placement of massive and symmetrical solid lead collars around the lateral circumference of the evacuated sphere. Great care is taken in the balanced placement of these collars so as to create only horizontal gravitational field vectors between the collars and the sphere and its contents. Any weight increase in the sphere after placement in this enhanced horizontal gravitational field should be attributable to the increased gravitational energy (and the associated increased mass) of the gravitized vacuum, since the symmetrical metal shell of the sphere should remain balanced in the gravitational field. Repeated measurements, with top-to-bottom inversions of the collar and sphere between measurements, should serve to null out inadvertent net vertical gravitational force contributions of the collar on the sphere. The magnitude of any confirmed weight and mass density increase can then be compared to the estimated average dark matter density of the Milky Way galaxy (see below).

\section{Summary and Discussion}

The gravitized vacuum is operationally defined in this paper and a hypothesis is proposed that all observations attributed to dark matter are predictable effects of the expected increased energy density of the gravitized vacuum.

A low-end estimate of the Milky Way average dark matter mass density can be made as follows: based upon the visible matter mass of approximately $2 \times 10^{41} \mathrm{~kg}$ ( $10^{11}$ solar masses) and a (at minimum) 5:1 galactic dark matter-to-visible matter ratio, the Milky Way dark matter is estimated to have a mass of approximately $10^{42} \mathrm{~kg}$; the Milky Way volume, assuming a disc of 100,000 light-years in diameter and an average disc thickness of 1000 light-years, is approximately $6.65 \times 10^{60}$ $\mathrm{m}^{3}$. Dividing the dark matter mass by the Milky Way volume gives a low-end average dark matter density estimate of approximately $1.5 \times 10^{-19} \mathrm{~kg} \cdot \mathrm{m}^{-3}$. The maximum value of the Milky Way dark matter-to-visible matter ratio could be considerably higher than 5:1, although the theoretical maximum is unknown to this author at the present time. 
Given the above average dark matter density estimate, it is remarkable how great the large-scale effects can be on gravitational lensing and galactic rotation curves. Obviously, the experimental apparatus of Figure 1 would not be able to detect a gravitized vacuum mass increase corresponding to this low density magnitude. However, as such, any reliably detectable gravitized vacuum mass density increase would be considerably above the Milky Way density estimate and therefore supportive of the dark matter theory presented herein.

\section{Conflicts of Interest}

The author declares no conflicts of interest regarding the publication of this paper.

\section{References}

[1] Milgrom, M. (1983) Astrophysical Journal, 270, 365-370. https://doi.org/10.1086/161130

[2] Kroupa, P., Pawlowski, M. and Milgrom, M. (2012) International Journal of Modern Physics D, 21, Article ID: 1230003. arXiv:1301.3907 https://doi.org/10.1142/S0218271812300030

[3] Verlinde, E. (2011) Journal of High Energy Physics, 4, 29-55. arXiv:1001.0785v1 [hep-th] https://doi.org/10.1007/JHEP04(2011)029

[4] Verlinde, E. (2016) Emergent Gravity and the Dark Universe. arXiv:1611.02269v2 [hep-th]

[5] Tatum, E.T. and Seshavatharam, U.V.S. (2018) Journal of Modern Physics, 9, 1568-1572. https://doi.org/10.4236/jmp.2018.98098

[6] Brouwer, M.M., et al. (2016) Monthly Notices of the Royal Astronomical Society, 446, 2547-2559. arXiv:1612.03034v2 [astro-ph.CO]

[7] Tatum, E.T. (2018) Journal of Modern Physics, 9, 1564-1567. https://doi.org/10.4236/jmp.2018.98097

[8] Hossenfelder, S. and McGough, S.S. (2018) Scientific American, 319, 37-43. https://doi.org/10.1038/scientificamerican0818-36

[9] Berezhiani, L. and Khoury, J. (2016). Theory of Dark Matter Superfluidity. arXiv:1507.01019v2 [astro-ph.CO] 